\title{
Null broadening adaptive beamforming based on covariance matrix reconstruction and similarity constraint
}

\author{
Junhui Qian*, Zishu He, Julan Xie and Yile Zhang
}

\begin{abstract}
In this paper, a procedure for the null broadening algorithm design with respect to the nonstationary interference is proposed. In contrast to previous works, we first impose nulls toward the regions of the nonstationary interference based on the reconstruction of the interference-plus-noise covariance matrix. Additionally, in order to provide a restriction on the shape of the beam pattern, a similarity constraint is enforced at the design stage. Then, the adaptive weight vector can be computed via maximizing a new signal-to-interference-plus-noise ratio (SINR) criterion subject to similarity constraint. Mathematically, the design original problem is expressed as a nonconvex fractional quadratically constrained quadratic programming (QCQP) problem with additional constraint, which can be converted into a convex optimisation problem by semidefinite programming (SDP) techniques. Finally, an optimal solution can be found by using the Charnes-Cooper transformation and the rank-one matrix decomposition theorem. Several numerical examples are performed to validate the performance of the proposed algorithm.
\end{abstract}

Keywords: Robust adaptive beamforming, Similarity constraint, Quadratically constrained quadratic programming, Semidefinite programming

\section{Introduction}

Adaptive beamforming has been one of the most significant research areas in array signal processing, which has been widely used in radar, sonar, wireless communications, and many other fields [1]. The aim of adaptive beamforming algorithm is to extract the desired signal and suppress the interference as well as noise at the array output simultaneously. However, the conventional beamforming method often suffers severe performance degradation because of some factors, such as small training snapshots and imprecise knowledge in many practical applications [2]. Thus, robust design techniques have been an active research topic $[3,4]$. During the past decade, various adaptive beamformers have been proposed based on different principles to improve the robustness against the desired signal imprecisely [5], such as signal-subspace projection technique, diagonal loading technique, and their variants [6, 7]; all of the methods are quite efficient in

${ }^{*}$ Correspondence: junhuiq123@163.com

School of Electronic Engineering, University of Electronic Science and

Technology of China, 2006 Xi Yuan Ave, West Hi-tech Zone, Chengdu, China enhancing the robustness of the beamformer. Recently, in order to remove the desired signal from the sample covariance matrix, spatial power spectrum sampling methods were proposed to achieve covariance matrix reconstruction $[8,9]$.

It is also well known that the adaptive beamforming is quite sensitive to the nonstationary interference. For the interference, the model parameters could be determined or randomized. Its waveform or distribution may change with the time or location, which can be caused by the interference quickly moving, antenna platform vibrating, and propagation channel varying, etc. Moreover, due to the limited computational resources, in many practical situations, continuously updating the optimal adaptive weight vector may be prohibitive. The resulting beamformer weights are frozen and used for the remainder of the frame despite changes in real-world scenario, which considerably degrade the output signal-to-interferenceplus-noise ratio (SINR) performance in nonstationary environments [10]. Especially for large aperture arrays, the perturbation of the interference location represents a serious problem because the directional pattern nulls of them 
are extremely sharp, and interferences may move out of the nulls very soon [11]; thus, the adaptive beamformer may degrade rapidly with the change of interference location. However, few of the above discussed robust methods could handle the problem of nonstationary interference.

The nulling broadening algorithms can solve such problems hereinabove and avoid the additional complexity of the adaptive weight vector continuously updating. In [12], the approach to robust beamforming, with null widening, imparts robustness into adaptive pattern by judicious choice of null placement and width through introducing the concept of a covariance matrix taper (CMT), while the performance degradation of the method is evident because the relative high sidelobe and the depth become somewhat shallower when the null width is broadened. Several null widening techniques based on matrix tapers have been proposed to overcome the pattern distortion resulting from the nonstationary interference $[13,14]$. Nevertheless, these methods are somewhat identical in essence and attain similar performance in output SINR. In [15], a beamforming framework has been proposed based on a set of beam pattern shaping constraints; this method enjoys adaptive interference-rejection capability and controls direct sidelobe, meanwhile, achieves robustness against steering direction errors with magnitude response constraints. However, these constraints consume the number of adaptive degrees of freedom (DOFs) to trade off the output SINR. The multiparametric quadratic programming for covariance matrix taper minimum variance distortionless response beamformer is proposed to resolve null broadening and sidelobe control problem in [16]. Nevertheless, the sidelobe domain constraint is obviously broadening the mainlobe beam pattern which decreases in array gain.

In this work, we develop a novel null broadening approach with respect to the nonstationary interference. The original problem is optimized to maximize the output SINR and to force a similarity constraint with a quiescent steering vector exhibiting some desirable properties. We attempt to reconstruct the interference-plus-noise covariance matrix in order to impose nulls toward the angular sectors of the nonstationary interference. Meanwhile, based on the similarity constraint, the proposed method can obtain lower sidelobe and well-maintained mainlobe of the beam pattern. The design problem is formulated as a fractional quadratically constrained quadratic programming (QCQP) problem [17]. The original nonconvex problem can be converted into a convex optimisation problem by semidefinite programming (SDP) techniques [18]. Finally, a global optimal solution can be found by using the Charnes-Cooper transformation and the rank-one matrix decomposition theorem $[19,20]$. As a result, the process imposes nulls towards the regions of the nonstationary interference adaptively. Meanwhile, it guarantees that the pattern is extremely close to the quiescent pattern through the similarity constraint; the output SINR can be improved substantially. Theoretical analysis and simulation results show that the proposed method provides excellent performance.

\section{Signal model}

Consider a uniform linear array (ULA) comprising $M$ antennas with interelement spacing $d$; the narrowband snapshot vector is simply modeled and can be expressed as

$$
\begin{aligned}
\mathbf{x}(t) & =\mathbf{x}_{s}(t)+\mathbf{x}_{i}(t)+\mathbf{n}(t) \\
& =s_{0}(t) \mathbf{a}\left(\theta_{0}\right)+\sum_{i=1}^{P} s_{i}(t) \mathbf{a}\left(\theta_{i}\right)+\mathbf{n}(t)
\end{aligned}
$$

where $t$ is the index of time. $\mathbf{x}_{s}(t), \mathbf{x}_{i}(t), \mathbf{n}(t)$ are the desired signal, interference, and noise components, respectively. $s_{0}(t), s_{i}(t)$ denote the desired signal waveform and interference signal waveform, respectively. Assume that the desired signal, interference, and noise are statistically independent with each other, and the interference is neither close to nor in the mainlobe beam region of the array. $\theta_{0}$ and $\theta_{i}(i \in\{1, \ldots, P\})$ denote the directions of arrival of the desired signal and the $i$ th interference, respectively. a $(\cdot)$ denotes the corresponding $M \times 1$ steering vector, which has the following general form

$$
\mathbf{a}(\theta)=\left[\begin{array}{llll}
1 e^{j 2 \pi d \sin \theta / \lambda} & \ldots e^{j 2 \pi(M-1) d \sin \theta / \lambda}
\end{array}\right]^{T}
$$

where $\lambda$ is the signal wavelength, $(\cdot)^{T}$ denotes the transpose operation. The adaptive beamformer output can be written as $\mathbf{y}(t)=\mathbf{w}^{H} \mathbf{x}(t)$, where $\mathbf{w}$ denotes the $M \times 1$ complex weight beamforming vectors and $(\cdot)^{H}$ stands for the Hermitian transpose. The weight vector can be found from the maximum of the output SINR

$$
\operatorname{SINR}=\frac{\mathbf{w}^{H} \mathbf{R}_{s} \mathbf{w}}{\mathbf{w}^{H} \mathbf{R}_{i n} \mathbf{w}}=\frac{\sigma_{0}^{2}\left|\mathbf{w}^{H} \mathbf{a}\left(\theta_{0}\right)\right|^{2}}{\mathbf{w}^{H} \mathbf{R}_{i n} \mathbf{w}}
$$

where $\sigma_{0}^{2} \triangleq \mathrm{E}\left\{\left|s_{0}(t)\right|^{2}\right\}$ denotes the desired signal power, $\mathbf{E}\{\cdot\}$ is an expectation operator, and $|\cdot|$ is an absolute operator. Assume $\sigma_{i}^{2}$ denotes the $i$ th interference power. $\mathbf{R}_{\mathbf{s}}=E\left\{\mathbf{x}_{s}(t) \mathbf{x}_{s}^{H}(t)\right\}$ and $\mathbf{R}_{i n}=$ $\mathrm{E}\left\{\left(\mathbf{x}_{i}(t)+\mathbf{n}(t)\right)\left(\mathbf{x}_{i}(t)+\mathbf{n}(t)\right)^{H}\right\}$ are the desired signal and the interference-plus-noise covariance matrix, respectively. Because all the signals are mutually uncorrelated, the theoretical covariance matrix of the array signal can be expressed as follows

$$
\begin{aligned}
\mathbf{R}_{\mathrm{x}} & =\mathbf{R}_{s}+\mathbf{R}_{i n} \\
& =\sigma_{0}^{2} \mathbf{a}\left(\theta_{0}\right) \mathbf{a}^{H}\left(\theta_{0}\right)+\sum_{i=1}^{P} \sigma_{i}^{2} \mathbf{a}\left(\theta_{i}\right) \mathbf{a}^{H}\left(\theta_{i}\right)+\sigma_{n}^{2} \mathbf{I}
\end{aligned}
$$


where $\mathbf{R}_{s}=\sigma_{0}^{2} \mathbf{a}\left(\theta_{0}\right) \mathbf{a}^{H}\left(\theta_{0}\right)$ and $\mathbf{R}_{i n}=\sum_{i=1}^{P} \sigma_{i}^{2} \mathbf{a}\left(\theta_{i}\right) \mathbf{a}^{H}\left(\theta_{i}\right)+$ $\sigma_{n}^{2} \mathbf{I}, \sigma_{n}^{2}$ and $\mathbf{I}$ represent the noise power and the identity matrix, respectively. In practical implementations, the theoretical covariance matrix is hard to be obtained, and it is usually replaced by the sample covariance matrix $\hat{\mathbf{R}}_{\mathrm{x}}$, which is calculated from the received signal vectors as follows

$$
\hat{\mathbf{R}}_{\mathbf{x}}=\frac{1}{N} \sum_{t=1}^{N} \mathbf{x}(t) \mathbf{x}^{H}(t)
$$

where $N$ is the number of snapshots. The beamforming weight vector $\mathbf{w}$ can be obtained via maximizing the problem (3), which is mathematically equivalent to maintaining a distortionless response towards the desired signal and minimizing the output interference-plus-noise power problem

$$
\min _{\mathbf{w}} \mathbf{w}^{H} \hat{\mathbf{R}}_{\mathbf{x}} \mathbf{w} \quad \text { s.t. } \quad \mathbf{w}^{H} \mathbf{a}\left(\theta_{0}\right)=1
$$

Then, the adaptive weight vector based on minimum variance distortionless response (MVDR) principle can be given by

$$
\mathbf{w}=\frac{\hat{\mathbf{R}}_{\mathbf{x}}^{-1} \mathbf{a}\left(\theta_{0}\right)}{\mathbf{a}^{H}\left(\theta_{0}\right) \hat{\mathbf{R}}_{\mathbf{x}}^{-1} \mathbf{a}\left(\theta_{0}\right)}
$$

It should be stated that $\hat{\mathbf{R}}_{\mathrm{x}}$ contains the desired signal component. Hence, the adaptive weight vector obtained by using $\hat{\mathbf{R}}_{\mathrm{x}}$ is worse than the one using the covariance matrix without any contribution from the desired signal. The maximization problem of SINR performance in (3) is equivalent to the minimum problem of the variance distortionless response criterion. In practical situations, the unexpected performance degradation of beamformer may emerge caused by interference nonstationarity. In order to measure the performance of the beamformer, the goal of improving the robustness of adaptive beamforming is to maximize the output SINR in the following section.

\section{Proposed algorithm}

As can be seen from (3), maximizing the SINR needs the exact knowledge of desired signal and interference. In this section, we focus on the nonstationary interference suppression problem, namely, the null broadening algorithm with interference-plus-noise covariance matrix reconstruction, and beam pattern similarity constraint is proposed on the condition that the exact knowledge of target parameters is known or previously estimated. When the interference moves quickly or the antenna platform vibrates, continuously updating of the adaptive weight vector can be prohibitive; the mismatching between adaptive weight and data occurs due to the change of interference locations. We design a beamformer that can flexibly adjust the null width and avoid the additional complexity of continuously updating. Meanwhile, it can enjoy low sidelobes and well-maintained mainlobe of the beam pattern at low snapshots.

\subsection{Proposed formulation}

The spatial power spectrum is mainly distributed the neighborhood of the angular sector in where the signal located. Thus, the interference covariance matrix reconstruction can be performed based on the Capon spectrum by integrating over the angular sector where the interference located. Let us consider the direction of the $i$ th interference under test is known to lie centered around $\theta_{i}$. The angular sector of the $i$ th interference can be expressed via the following formula

$$
\boldsymbol{\Phi}_{i}=\left[\theta_{i}-\Delta \theta_{i}, \theta_{i}+\Delta \theta_{i}\right], i=1, \ldots, P
$$

where $\Delta \theta_{i}$ indicates the level of angular uncertainty. In practical situation, $\Delta \theta_{i}$ can be obtained using low resolution direction finding methods [21]. Thus, we will obtain the beamformer weights by solving the following optimization problem

$$
\underset{\mathbf{w}, \theta_{i} \in \boldsymbol{\Phi}_{i}, i=1, \ldots, P}{\operatorname{maximize}} \frac{\sigma_{0}^{2}\left|\mathbf{w}^{H} \mathbf{a}\left(\theta_{0}\right)\right|^{2}}{\mathbf{w}^{H} \sum_{i=1}^{P} \sigma_{i}^{2} \mathbf{a}\left(\theta_{i}\right) \mathbf{a}^{H}\left(\theta_{i}\right) \mathbf{w}+\sigma_{n}^{2} \mathbf{w}^{H} \mathbf{w}}
$$

In this correspondence, we attempt to construct the interference-plus-noise covariance matrix in order to impose nulls towards the angular sectors of the nonstationary interference. For the covariance matrix, we first need to know the steering vectors and powers of all interferences, and the noise power. According to the robust adaptive beamforming technique in [8], the interferenceplus-noise covariance matrix $\mathbf{R}_{i n}$ can be reconstructed as

$$
\left.\tilde{\mathbf{R}}\right|_{i n}=\int_{\tilde{\Theta}} \hat{P}(\theta) \mathbf{a}(\theta) \mathbf{a}^{H}(\theta) d \theta
$$

where $\bar{\Theta}$ is the angular region excluding the desired signal region $\Theta$ in the whole spatial domain. $\hat{P}(\theta)$ denotes the Capon spectrum as an estimate of the spatial power spectrum over all possible directions, which is given as

$$
\hat{P}(\theta)=\frac{1}{\mathbf{a}^{H}(\theta) \hat{\mathbf{R}}_{\mathbf{x}}^{-1} \mathbf{a}(\theta)}
$$

To achieve the goal of the interference suppression, deep null notches should be formed towards the spatial directions, and in order to make sure that each steering vector of the nonstationary interference can be covered by the angular sector of the interference, the power of the $i$ th interference region can be modified as

$$
\sigma_{i}^{2} \approx \max \left\{\frac{1}{\mathbf{a}^{H}(\theta) \hat{\mathbf{R}}_{\mathbf{x}}^{-1} \mathbf{a}(\theta)} \mid \theta \in\left[\theta_{i}-\Delta \theta_{i}, \theta_{i}+\Delta \theta_{i}\right]\right\}
$$


The convergence of (12) can easily estimate the $i$ th interference power using the Capon spectral estimator in the angular sector $\boldsymbol{\Phi}_{i}$. Thus, the $i$ th interference covariance matrix can be formulated as

$$
\mathbf{r}\left(\theta_{i}\right)=\int_{\theta \in \boldsymbol{\Phi}_{i}} \frac{\mathbf{a}(\theta) \mathbf{a}^{H}(\theta)}{\min \left\{\mathbf{a}^{H}(\theta) \hat{\mathbf{R}}_{\mathrm{x}}^{-1} \mathbf{a}(\theta)\right\}} d \theta
$$

Let $\theta_{i l} \in \boldsymbol{\Phi}_{i}$ be a chosen grid that approximates the $i$ th interference area by using a finite number of angles, which can be expressed as $\boldsymbol{\Phi}_{i}=$ $\left\{\theta_{i 1} \ldots \theta_{i l} \ldots \theta_{i L}\right\}$, where $i L$ denotes the number of sampled values. The convergence of (12) can be recast as $\tilde{\sigma}_{i}^{2}=$ $\max \left\{\hat{P}\left(\theta_{i 1}\right), \ldots \ldots, \hat{P}\left(\theta_{i L}\right)\right\}$, which means that the interference covariance matrix is reconstructed to create null notches towards the sampled points in angular sectors of the nonstationary interference, and thus, the wide null notches can be formed. Then the $i$ th interference region covariance matrix can be calculated approximately as

$$
\tilde{\mathbf{r}}\left(\theta_{i}\right) \approx \tilde{\sigma}_{i}^{2} \sum_{i l=1}^{i L} \mathbf{a}\left(\theta_{i l}\right) \mathbf{a}^{H}\left(\theta_{i l}\right)
$$

The convergence of (14) means that the power of the neighborhood of the $i$ th interference is modeled as the same level $\tilde{\sigma}_{i}^{2}$ in the angular sector $\boldsymbol{\Phi}_{i}$. It is known that the noise subspace can be found by performing eigendecomposition on $\hat{\mathbf{R}}_{\mathrm{x}}$; nevertheless, the multiplicity of the noise eigenvalues cannot be accessed. Thus, the noise power can be approximately estimated by the average value of the noise eigenvalues as follows

$$
\tilde{\sigma}_{n}^{2}=1 /(M-P-1) \sum_{i=P+2}^{M} \lambda_{i}\left(\hat{\mathbf{R}}_{\mathrm{x}}\right)
$$

where $\lambda_{i}$ denotes the $i$ th eigenvalue of matrix $\hat{\mathbf{R}}_{\mathrm{x}}$. Thus, the beamformer weights can be obtained by solving the following optimization problem

$$
\underset{\mathbf{w}}{\operatorname{maximize}} \frac{\sigma_{0}^{2}\left|\mathbf{w}^{H} \mathbf{a}\left(\theta_{0}\right)\right|^{2}}{\mathbf{w}^{H} \sum_{i=1}^{P} \tilde{\sigma}_{i}^{2} \sum_{i l=1}^{i L} \mathbf{a}\left(\theta_{i l}\right) \mathbf{a}^{H}\left(\theta_{i l}\right) \mathbf{w}+\tilde{\sigma}_{n}^{2} \mathbf{w}^{H} \mathbf{w}}
$$

In addition to the aforementioned constraint, one can also consider other types of constraints that reinforce the beamformer to be not too different from a desired beamformer. As noted in $[15,16]$, the resulting broadening null technology may cause mainlobe distortion, sidelobe elevation, and array degradation. Therefore, additional constraints are necessary in the beamformer design problem. Usually, the quiescent weight vector is designed to produce quiescent beam patterns with desired mainlobe beamwidth and low peak sidelobes. To remedy the beam pattern distortion caused by adaptive processing, we need to reduce the perturbation of adaptive weights to the quiescent weight settings. Use the similar idea in waveform design [22]; a similarity constraint uses a known waveform as a benchmark, which allows the designed waveform to exhibit other desired waveform properties. In order to provide a control on the shape of the beam pattern, we can formulate the similarity constraint as

$$
\left\|\mathbf{w}-\mathbf{w}_{q}\right\|^{2} \leq \xi^{2}
$$

where $\mathbf{w}_{q}$ is the weighted quiescent steering vector. $\|\cdot\|$ denotes the Euclidean norm of a vector or the Frobenius norm of a matrix. For the specific signal direction $\theta_{0}, \mathbf{w}_{q}$ is the steering vector of the desired signal. $\xi$ is a tunable positive parameter ruling the extent of the similarity. In general, $\xi$ is a small value which satisfies $\xi<\left\|\mathbf{w}_{q}\right\|$ (to guarantee a nonzero solution). The constraint is used to control and mean-squared error between the adaptive and desired quiescent beam pattern [23]. Finally, the design problem can be formulated as the following constrained optimization problem

$$
p_{1}\left\{\begin{array}{l}
\underset{\mathbf{w}}{\operatorname{maximize}} \frac{\sigma_{0}^{2}\left|\mathbf{w}^{H} \mathbf{a}\left(\theta_{0}\right)\right|^{2}}{\mathbf{w}^{H} \sum_{i=1}^{P} \tilde{\mathbf{r}}\left(\theta_{i}\right) \mathbf{w}+\tilde{\sigma}_{n}^{2} \mathbf{w}^{H} \mathbf{w}} \\
\text { s.t. }\left\|\mathbf{w}-\mathbf{w}_{q}\right\|^{2} \leq \xi^{2}
\end{array}\right.
$$

It is worth observing that $p_{1}$ is a nonconvex fractional quadratic optimization problem; generally, such problem is NP-hard. However, this designed original problem can be solved using semidefinite relaxation (SDR) technique. Resorting to the SDR framework [24], now, we aim to design an algorithm able to find in polynomial time solutions with quality guarantee to the NP-hard problem.

\subsection{Solution to the optimization problem}

In this subsection, we devise a novel method to tackle the nonconvex fractional quadratic optimization problem $p_{1}$. As revealed by the analysis above, the interference-plusnoise covariance matrix can be computed as

$$
\tilde{\mathbf{R}}_{i n}=\sum_{i=1}^{P} \tilde{\mathbf{r}}\left(\theta_{i}\right)+\tilde{\sigma}_{n}^{2} \mathbf{I}
$$

It is evident that the original nonconvex design problem $p_{1}$ can be transformed to the following equivalent homogenous reformulation

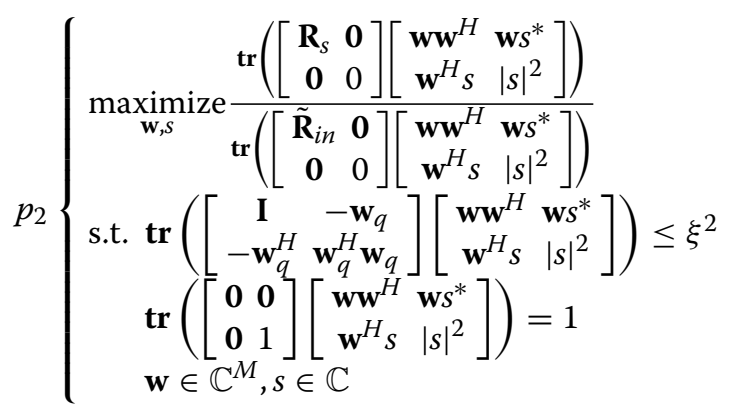


where $s^{*}$ is the conjugate of $s, \operatorname{tr}(\mathbf{A})$ represents the trace of matrix A. Precisely, $p_{1}$ and $p_{2}$ have the same optimal values, i.e., $v\left(p_{1}\right)=v\left(p_{2}\right)$. Next, we can solve $p_{2}$ in order to get an optimal solution to $p_{1}$. By applying the SDP relaxation procedure to this fractional QCQP $p_{2}$, we can obtain the following problem

$$
p_{3}\left\{\begin{array}{c}
\underset{\mathbf{G}}{\operatorname{maximize}} \frac{\operatorname{tr}\left(\mathbf{Q}_{0} \mathbf{G}\right)}{\operatorname{tr}\left(\mathbf{Q}_{1} \mathbf{G}\right)} \\
\text { s.t. } \operatorname{tr}\left(\mathbf{Q}_{2} \mathbf{G}\right) \leq \xi^{2} \\
\operatorname{tr}\left(\mathbf{Q}_{3} \mathbf{G}\right)=1 \\
\mathbf{G} \succeq \mathbf{0}
\end{array}\right.
$$

We use $\mathbf{A} \succeq 0$ to indicate that $\mathbf{A}$ is a positive semidefinite (PSD) matrix. Here, the matrix parameters are defined as follows

$$
\mathbf{Q}_{0}=\left[\begin{array}{cc}
\mathbf{R}_{s} & 0 \\
\mathbf{0} & 0
\end{array}\right], \mathbf{Q}_{1}=\left[\begin{array}{cc}
\tilde{\mathbf{R}}_{\text {in }} & \mathbf{0} \\
\mathbf{0} & 0
\end{array}\right], \mathbf{Q}_{2}=\left[\begin{array}{cc}
\mathbf{I} & -\mathbf{w}_{q} \\
-\mathbf{w}_{q}^{H} & \mathbf{w}_{q}^{H} \mathbf{w}_{q}
\end{array}\right]
$$

and

$$
\mathbf{Q}_{3}=\left[\begin{array}{ll}
\mathbf{0} & 0 \\
\mathbf{0} & 1
\end{array}\right], \mathbf{G}=\left[\begin{array}{ll}
\mathbf{w} \mathbf{w}^{H} & \mathbf{w} s^{*} \\
\mathbf{w}^{H} s & |s|^{2}
\end{array}\right]
$$

The SDR problem $p_{3}$ does not immediately lead to an $\mathrm{SDP}$. That is to say, the SDR problem $p_{3}$ is a quasi-convex problem, due to the linear fractional structure of its objective function. In order to solve this quasi-convex problem, the fractional problem $p_{3}$ can be iteratively solved by the bisection search. Here we propose an optimal solution by using the idea of Charnes-Cooper transformation and the rank-one matrix decomposition theorem. Let us define the transformed variable $\mathbf{X}=\gamma \mathbf{G}$, where $\gamma \geq 0$ complies with $\operatorname{tr}\left(\mathbf{Q}_{1} \mathbf{X}\right)=1$, applying the Charnes-Cooper transform, we can obtain the solution of $p_{3}$ by solving the following SDP problem

$$
p_{4} \begin{cases}\underset{\mathbf{X}, \gamma}{\operatorname{maximizetr}}\left(\mathbf{Q}_{0} \mathbf{X}\right) \\ \text { s.t. } & \operatorname{tr}\left(\mathbf{Q}_{1} \mathbf{X}\right)=1 \\ & \operatorname{tr}\left(\mathbf{Q}_{2} \mathbf{X}\right) \leq \gamma \xi^{2} \\ & \operatorname{tr}\left(\mathbf{Q}_{3} \mathbf{G}\right)=\gamma \\ & \mathbf{X} \geq \mathbf{0}, \gamma \geq 0\end{cases}
$$

We exploit the Charnes-Cooper variable transformation and a specific rank-one matrix decomposition theorem [25], which is cited as the following lemma.

Lemma 1 Let $\mathbf{X}$ be a non-zero $M \times M(M \geq 3)$ complex Hermitian positive semidefinite matrix and $\mathbf{A}_{i}$ be Hermitian matrix, $i=1,2,3,4$, and suppose that $\left(\operatorname{tr}\left(\mathbf{Y A}_{1}\right), \operatorname{tr}\left(\mathbf{Y A}_{2}\right), \operatorname{tr}\left(\mathbf{Y A}_{3}\right), \operatorname{tr}\left(\mathbf{Y A}_{4}\right)\right) \neq(0,0,0,0)$ for any non-zero complex Hermitian positive semidefinite matrix $\mathbf{Y}$ of size $M \times M$. Then,

- if rank $(\mathbf{X}) \geq 3$, one can find, in polynomial time, a rank-one matrix $\mathbf{x x}^{H}$ such that $\mathbf{x}$ (synthetically denoted as $\left.\mathbf{x}=\mathcal{D}_{1}\left(\mathbf{X}, \mathbf{A}_{1}, \mathbf{A}_{2}, \mathbf{A}_{3}, \mathbf{A}_{4}\right)\right)$ is in range $(\mathbf{X})$, and

$$
\mathbf{x}^{H} \mathbf{A}_{i} \mathbf{x}=\operatorname{tr}\left(\mathbf{X} \mathbf{A}_{i}\right), i=1,2,3,4
$$

- if $\operatorname{rank}(\mathbf{X})=2$, for any $\mathbf{z}$ not in the range space of $\mathbf{X}$, one can find a rank-one matrix $\mathbf{x x}^{H}$ such that $\mathbf{x}$ (synthetically denoted as $\left.\mathbf{x}=\mathcal{D}_{2}\left(\mathbf{X}, \mathbf{A}_{1}, \mathbf{A}_{2}, \mathbf{A}_{3}, \mathbf{A}_{4}\right)\right)$ is in the linear subspace spanned by range $\{\mathbf{z}\} \cup \operatorname{range}(\mathbf{X})$, and

$$
\mathbf{x}^{H} \mathbf{A}_{i} \mathbf{x}=\operatorname{tr}\left(\mathbf{X A}_{i}\right), i=1,2,3,4
$$

Summarizing, an optimal solution $\mathbf{w}^{\#}$ of $p_{1}$ can be obtained according to Algorithm 1.

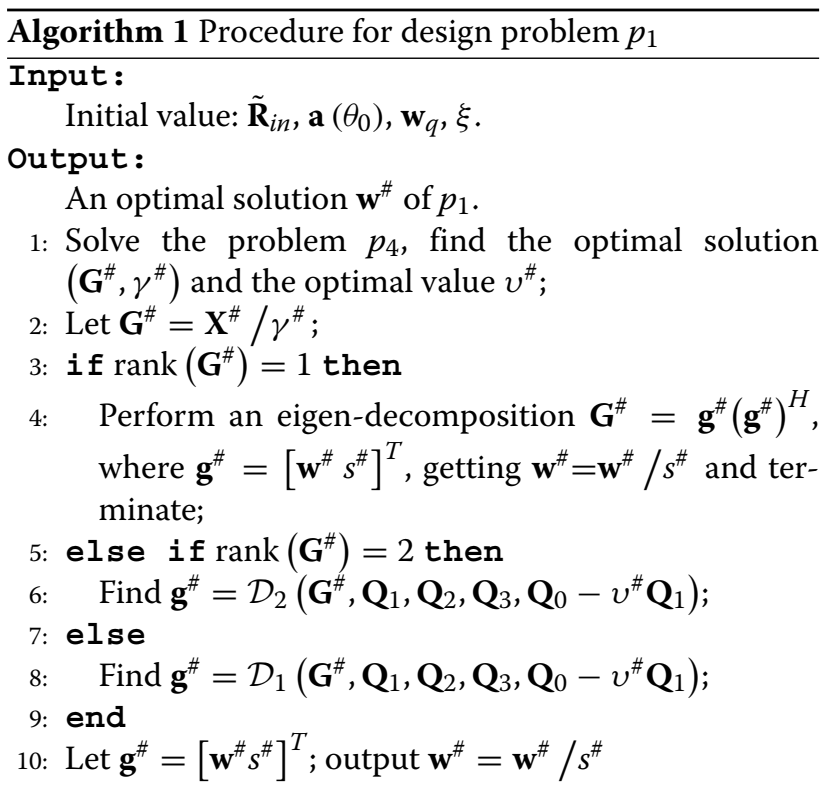

In this paper, we propose a novel null broadening algorithm design with respect to the nonstationary interference, and the design avoids the additional complexity of the weight vector continuously updating. Consider one similarity constraint with a quiescent steering vector exhibiting some desirable properties. Indeed, the proposed framework also achieves wide nulls by trading off some degrees of freedom (DOFs) of the array. In general, a beamformer is expected when more constraints are imposed but they consume more array's DOFs and tradeoff the output SINR. For the complex-valued problem, if the number of constraints is not more than 3 , the homogeneous QCQP can be efficiently solved in polynomial time [26]; in other words, the SDP relaxation is tight and hence, a global optimal solution of the original problem is achievable. Note that estimating structured covariance matrices is of particular interest in a variety of applications. The interference covariance matrix is reconstructed based on the Capon spectrum in (11), generally, not an optimal method [27]. The maximum likelihood approach is a good representative of the covariance matrix estimation techniques, which could yield better estimation performance [28-30]. Future work will concentrate on 
the advanced estimator design with respect to covariance matrix estimation.

In our algorithm, the main complexity lies in the interference-plus-noise covariance matrix reconstruction problem and the nonconvex fractional QCQP problem. The computation complexity of the noise power is dominated by the eigenvalue decomposition of $\hat{\mathbf{R}}_{\mathrm{x}}$, which is $O\left(M^{3}\right)$. The computation complexity of the interference covariance matrix reconstruction is $O\left(M^{2} J\right)$, where $J$ is the number of sampled points in the angular sector of the nonstationary interference. Generally speaking, $J$ is larger than $M$ that can improve the performance significantly. The computational complexity of Algorithm 1 is dominated by the solution of the SDP, at a complexity cost of $O\left(M^{4.5} \log (1 / \eta)\right.$ ) (where $\eta>0$ is a prescribed accuracy, e.g., see [31]), and the complexity of the rank-one decomposition requires operations $O\left(M^{3}\right)$.

\section{Simulation}

In this section, in order to evaluate the efficiency of the proposed algorithm, several simulated examples are performed to illustrate the results of our arguments on the nonstationary interference. Consider a uniform linear array with $M=20$ omnidirectional antenna elements spaced one-half wavelength apart. The additive noise is modeled as a complex Gaussian zero-mean spatially and temporally white process with unit variance in each sensor. Assume that the exact knowledge of desired signal parameters is known or previously estimated to the array receiver, which is set to be $0^{\circ}$, and the signal-to-noiseratio (SNR) is set to be $0 \mathrm{~dB}$. Consider applications where frozen weights with nonstationary interference are used. Two equal-power interference signals are presumed on the array from the directions of $-20^{\circ}$ and $30^{\circ}$, respectively. The interference-to-noise ratio (INR) in each sensor is equal to $35 \mathrm{~dB}$. For each scenario, 200 Monte Carlo runs are performed.

The proposed method is investigated and compared with the sample matrix inverse (SMI) method, the Mailloux-Zatman covariance matrix taper (MZT) notchwidening approach in [12], the beamforming framework based on the use of a set of beam pattern shaping constraints (BSC) in [15], and the multiparametric programming (CMT-MP-QP) method in [16]. In [15], the response ripple of the mainlobe region is set as $1.2 \mathrm{~dB}$ and a fixed mainlobe width of $6^{\circ}$ is used. The peak sidelobe levels are kept below $-25 \mathrm{~dB}$ and the sidelobe beam pattern area is given by $\left[-90^{\circ},-10^{\circ}\right] \cup\left[10^{\circ}, 90^{\circ}\right]$ in BSC and CMTMP-QP methods. The null levels of beam patterns are set below $-60 \mathrm{~dB}$ in BSC, CMT-MP-QP, and proposed methods. Without loss of generality, the similar factor $\xi$ is set to be $0.2 *\left\|\mathbf{w}_{q}\right\|$ (i.e., $\xi=0.2 * M$ ) in proposed method.
Example 1: beam pattern of beamformers. In the first simulation, we test the performance of adaptive beamforming in nonstationary interference environments, and the quiescent array pattern (QUI) is provided for comparison. The number of snapshots is 200. After the frozen weights are applied, a scenario with the random look direction mismatch of the nonstationary interference is considered. Assume the interferences move with their directions changes from $[-4,4]$. That is to say, the directions of the two interferences are distributed in $[-24,-16]$ and $[26,34]$, respectively. Then, the taper parameter equals 0.05 in MZT method and CMT-MP-QP method to obtain approximate beam pattern null width of $8^{\circ}$. The widths of interference nulls are set as $8^{\circ}$ and a uniform grid is used to obtain the angles in BSC and proposed methods. The corresponding results are shown in Fig. 1. It is found that the SMI method is not able to broaden the interference nulls and it has very high sidelobe level, while other methods are robust against the nonstationary interference. It is shown that the proposed technique can create wide nulls in the nonstationary interference regions, and the pattern is very close to the quiescent pattern. However, the MZT method suffers from performance degradation with the interference nulls becoming higher and sidelobe elevation. In BSC and CMT-MP-QP methods, deep notches can be formed and maintain a distortionless response for the desired signal, which also achieves lower sidelobe level and wide mainlobe.

Example 2: SINR versus the number of snapshots. In this example, the SINR performance of these methods is tested versus the number of training snapshots, and the optimal performance (OPT) is provided for comparison. The other parameters remain the same as example 1 except the number of snapshots. From the result shown in Fig. 2, the algorithms for nulling broadening can obtain high output SINR and fast convergence rate and much better than the SMI algorithm. The SMI beamformer is sensitive to the nonstationary interference and requires a large of number snapshots to converge. It can be observed that the proposed algorithm performs best among the tested beamformers. The performance of the BSC method is very close to that of the CMT-MP-QP method due to the similar multiparametric quadratic constraint programming. Although the MZT method shows fast convergence rate, it suffers from performance degradation mainly due to the sidelobe elevation.

Example 3: SINR versus interference mismatch angle. In the third example, the output SINRs versus the look direction error of the nonstationary interference are investigated among the OPT, SMI, MZT, BSC, CMTMP-QP, and proposed methods. All parameters are as in example 1 except the mismatch angle of interference, and the corresponding results are shown in Fig. 3. As expected, a wider range of the nonstationary interference leads to 


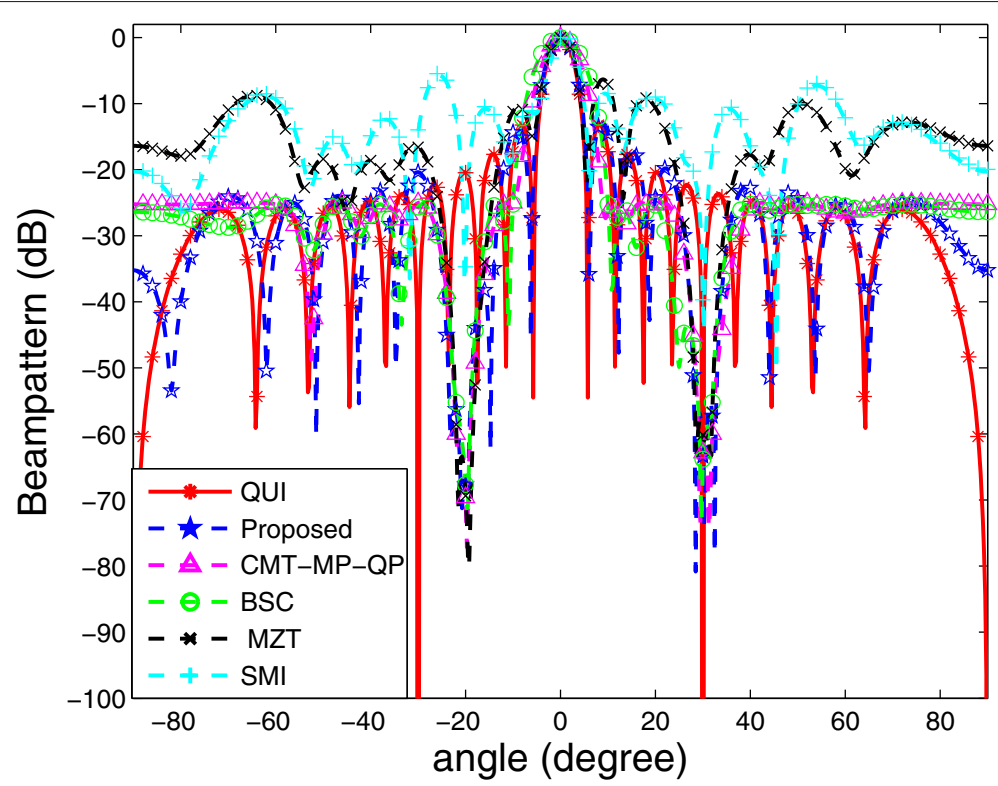

Fig. 1 Beam pattern of beamformers

a worse SINR. One can observe that the SMI method is quite extensive to the uncertainty and results in dramatic performance degradation, while the performances of other methods tend to stabilize with the mismatched angle range varying, which means that the nulling broadening algorithm can effectively suppress the nonstationary interference and avoid the additional complexity of the adaptive weight vector continuously updating. It is found that the proposed algorithm has the best performance among these adaptive beamforming algorithms. Therefore, the proposed beamforming algorithm outperforms other methods.

Example 4: the effect of the similarity constraint. In this section, we analyze the effect of the similarity parameter $\xi$ on the beam pattern of beamformer. Some values of the similarity parameter $\xi \in\{0.1 * M, 0.4 * M, 0.8 * M\}$ and other parameters remain the same as example 1 . The corresponding results are plotted in Fig. 4. As expected,

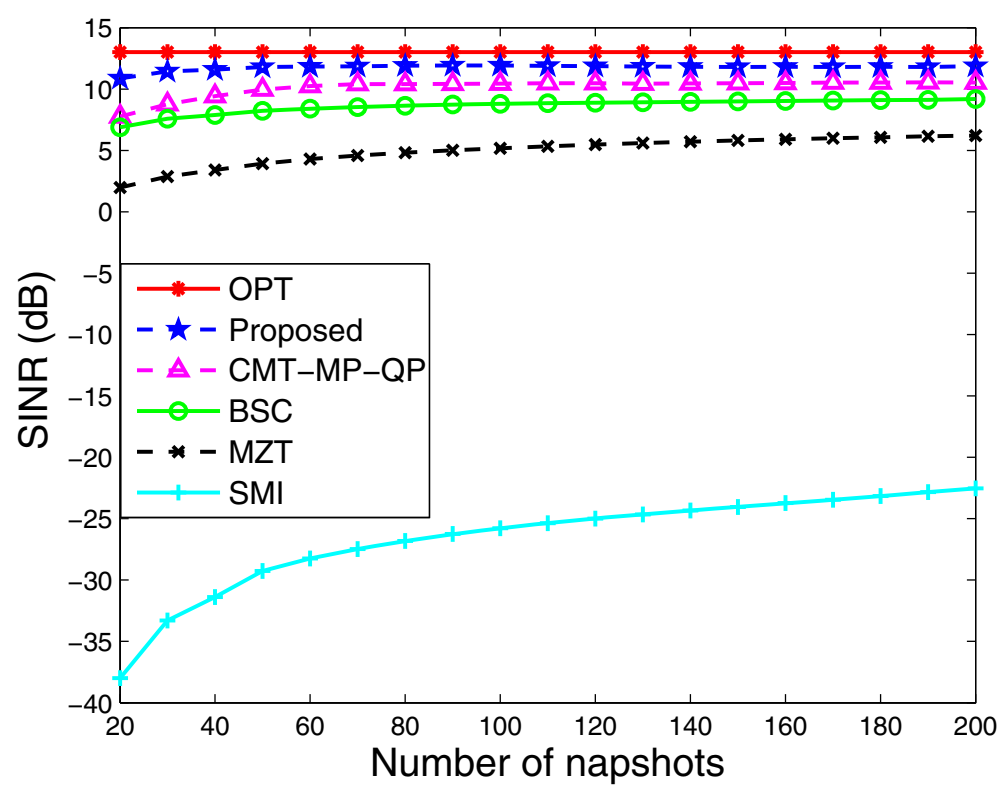

Fig. 2 SINR versus the number of snapshots 


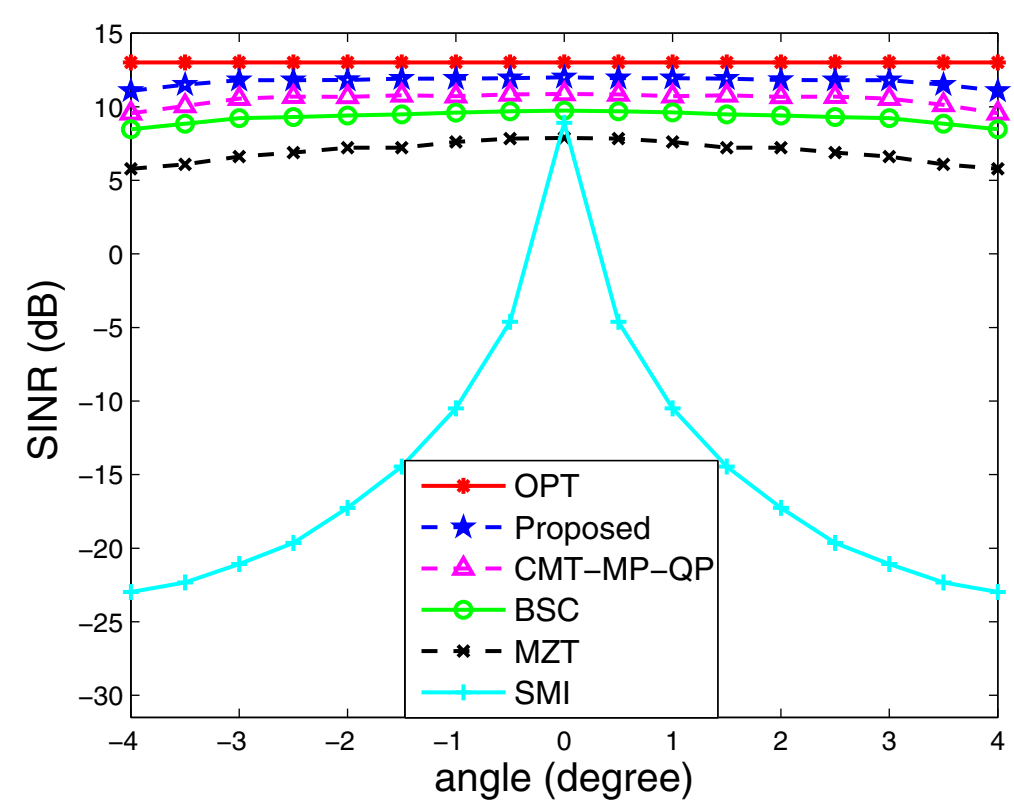

Fig. 3 SINR versus interference mismatch angle

the similarity constraint can effectively mitigate the beam pattern distortion resulting from the finite sample size or nonstationary interference. Additionally, when $\xi$ is smaller, the pattern is more similar to the quiescent pattern. However, as $\xi$ decreases, the null depths increase due to the similarity constraint. It should be noted that when $\xi$ converge towards 0 , the designed adaptive weight vector $\mathbf{w}$ is approximately equal to the quiescent weight vector $\mathbf{w}_{q}$, i.e., the beam pattern and the reference QUI are almost same, but with the notch vanishing. As such, the similarity parameter $\xi$ selected depends on the current scenario and several system parameters.

Example 5: SINR versus SNR for desired signal look direction mismatch. In the previous simulations, we consider only the perturbation of the interference location occurs. Although the proposed method is designed for solving only the interference mismatch problem, in this example, a desired signal look direction mismatch is

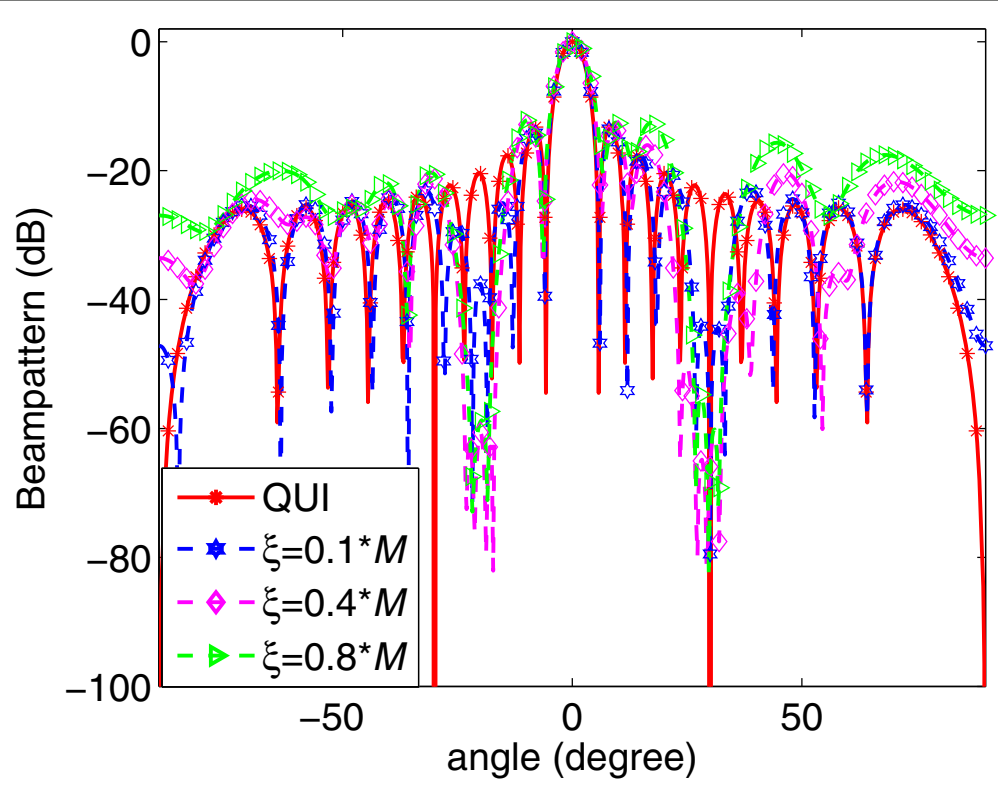

Fig. 4 The effect of similarity parameter on adaptive beamformer 


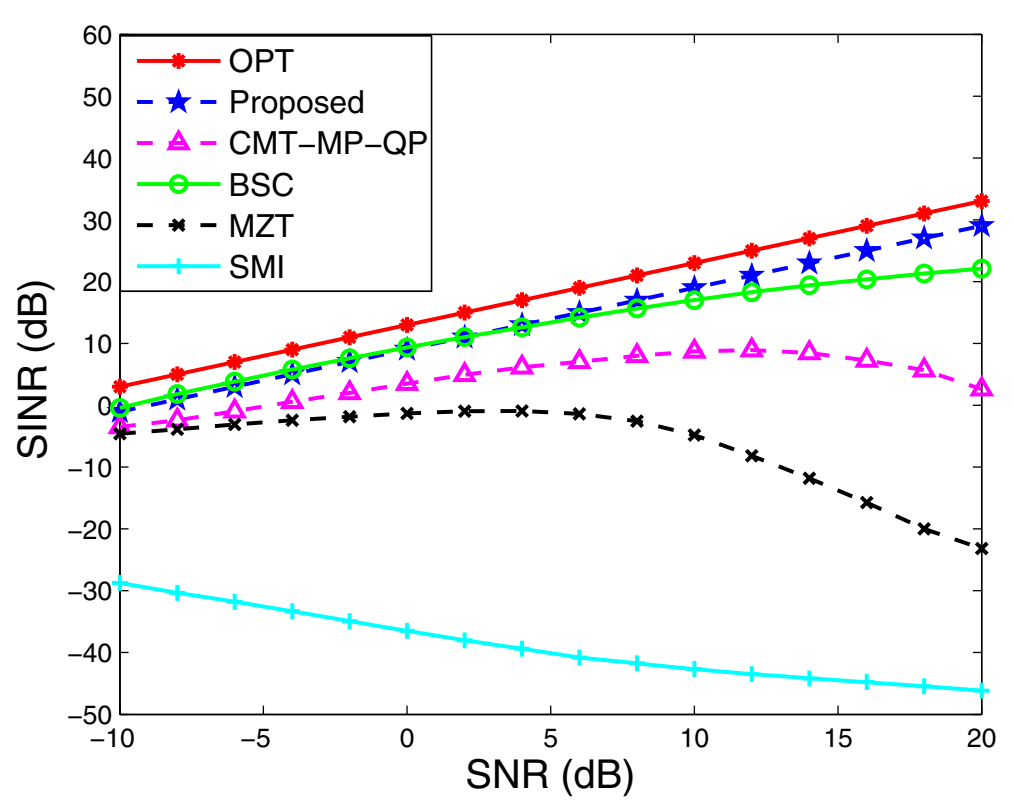

Fig. 5 SINR versus SNR for desired signal look direction mismatch

studied. All other parameters are chosen as before except the SNR. After frozen weights are used, the assumed arrival angle is $0^{\circ}$, but the actual arrival angle is $1.5^{\circ}$. The SINRs are compared for different SNRs ranging from -10 to $20 \mathrm{~dB}$. Note that the desired signal component is not present in the reconstructed interference-plus-noise covariance matrix, while the sample covariance matrix of other methods are corrupted by the desired signal. In Fig. 5 , it is clear that the proposed beamformer can obtain good output SINR in a large range of SNR, which is not sensitive to the power of the desired signal, thus, it implies that the proposed method reinforces the desired signal at the same time. The BSC beamformer maintains steady SINR with the SNR varying. In addition, due to the presence of the desired signal in training data, the output SINR of the BSC beamformer degrades slightly as SNR becomes larger. It can be observed that both the MZT and the CMT-MP-QP methods are affected severely by the desired signal look direction mismatch. The performance of the SMI beamformer is even worse as it cannot resolve the desired signal and interference mismatches. This example shows that the proposed method has unexpected good performance compared to other robust methods when a direction mismatches occurs. We believe that the proposed algorithm is a good candidate for robust beamforming.

\section{Conclusions}

We have discussed the problem of beamforming for the nonstationary interference; the goal of the proposed method is to achieve interference suppression algorithm design and avoid the additional complexity of the weight vector continuously updating. Compared to the previous algorithms, the proposed method is stable and effective for the interference nonstationarity. A nonconvex formulation for such optimization problem can be solved using SDR technique, Charnes-Cooper transformation, and rank-one matrix decomposition theorem. The proposed approach optimizes the performance via maximizing a new SINR criterion while maintains a distortionless response towards the direction of the desired signal, and deep null notches are formed towards the spatial directions of the interferences. The simulation results have demonstrated that the proposed beamformer can achieve a better performance, and it can be foreseen that this field will keep benefiting from advances in convex optimization theory. The covariance matrix reconstruction method brings some new ideas for interference suppression especially for the large aperture arrays.

\section{Acknowledgements}

The authors would like to express their deep appreciation to the reviewers who provided very useful criticism and many insightful remarks. This work is supported by the National Natural Science Foundation of China under Grants 61371184 and 61401062.

\section{Competing interests}

The authors declare that they have no competing interests.

Received: 1 August 2016 Accepted: 17 December 2016

Published online: 03 January 2017

References

1. RA Monzingo, TW Miller, Introduction to adaptive arrays. SciTech Publishing. (John Wiley \& Sons, New York, 1980) 
2. SA Vorobyov, AB Gershman, Z Luo, Robust adaptive beamforming using worst-case performance optimization: a solution to the signal mismatch problem. IEEE Trans. Signal Process. 51(2), 313-324 (2003)

3. A Aubry, V Carotenuto, A De Maio, New results on generalized fractional programming problems with toeplitz quadratics. IEEE Signal Process. Lett. 23(6), 848-852 (2016)

4. A Aubry, A De Maio, Y Huang, M Piezzo, Robust design of radar doppler filters. IEEE Trans. Signal Process. 64(22), 5848-5860 (2016)

5. J Li, P Stoica, Robust adaptive beamforming, vol. 88. (John Wiley \& Sons, New York, 2005)

6. J Zhuang, P Huang, Robust adaptive array beamforming with subspace steering vector uncertainties. Signal Process. Lett. IEEE. 19(12), 785-788 (2012)

7. J Li, P Stoica, Z Wang, On robust capon beamforming and diagonal loading. IEEE Trans. Signal Process. 51(7), 1702-1715 (2003)

8. Y Gu, A Leshem, Robust adaptive beamforming based on interference covariance matrix reconstruction and steering vector estimation. IEEE Trans. Signal Process. 60(7), 3881-3885 (2012)

9. J Xie, $\mathrm{H} \mathrm{Li}, \mathrm{Z} \mathrm{He}, \mathrm{C} \mathrm{Li}$, A robust adaptive beamforming method based on the matrix reconstruction against a large doa mismatch. EURASIP J. Adv. Signal Process. 2014(1), 1-10 (2014)

10. AB Gershman, GV Serebryakov, JF Bohme, Constrained hung-turner adaptive beam-forming algorithm with additional robustness to wideband and moving jammers. IEEE Trans. Antennas Propag. 44(3), 361-367 (1996)

11. BD Jeffs, KF Warnick, Bias corrected PSD estimation for an adaptive array with moving interference. IEEE Trans. Signal Process. 56(7), 3108-3121 (2008)

12. JR Guerci, Theory and application of covariance matrix tapers for robust adaptive beamforming. IEEE Trans. Signal Process. 47(4), 977-985 (1999)

13. RF Li, YL Wang, SH Wan, Research on adapted pattern null widening techniques. Modern Radar. 2(2), 42-45 (2003)

14. H Song, WA Kuperman, WS Hodgkiss, P Gerstoft, J Kim, Null broadening with snapshot-deficient covariance matrices in passive sonar. IEEE J. Ocean. Eng. 28(2), 250-261 (2003)

15. SE Nai, W Ser, ZL Yu, S Rahardja, A robust adaptive beamforming framework with beampattern shaping constraints. IEEE Trans. Antennas Propag. 57(7), 2198-2203 (2009)

16. FL Liu, PP Chen, JK Wang, P Lu, Null broadening and sidelobe control method based on multi-parametric quadratic programming. J. Northeas. Univ. 33(11), 1559-1562 (2012)

17. S Boyd, L Vandenberghe, Convex optimization. (Cambridge University Press, Cambridge, 2004), p. 1859

18. Z Luo, WK Ma, MC So, Y Ye, S Zhang, Semidefinite relaxation of quadratic optimization problems. IEEE Signal Proc. Mag. 27(3), 20-34 (2010)

19. A De Maio, Y Huang, DP Palomar, S Zhang, A Farina, Fractional QCQP with applications in ML steering direction estimation for radar detection. IEEE Trans. Signal Process. 59(1), 172-185 (2011)

20. A Aubry, A DeMaio, A Farina, M Wicks, Knowledge-aided (potentially cognitive) transmit signal and receive filter design in signal-dependent clutter. IEEE Trans. Aerosp. Electron. Syst. 49(1), 93-117 (2013)

21. L Huang, J Zhang, XXu, Z Ye, Robust adaptive beamforming with a novel interference-plus-noise covariance matrix reconstruction method. IEEE Trans. Signal Process. 63(7), 1643-1650 (2015)

22. G Cui, H Li, M Rangaswamy, Mimo radar waveform design with constant modulus and similarity constraints. IEEE Trans. Signal Process. 62(2), 343-353 (2014)

23. HLV Trees, Optimum array processing: part IV of detection, estimation, and modulation theory. (John Wiley \& Sons, New York, 2002)

24. G Wang, A semidefinite relaxation method for energy-based source localization in sensor networks. IEEE Trans. Veh. Technol. 60(5), 2293-2301 (2011)

25. W Ai, Y Huang, $\mathrm{S}$ Zhang, New results on hermitian matrix rank-one decomposition. Math. Program. 128(1-2), 253-283 (2011)

26. Y Huang, DP Palomar, Randomized algorithms for optimal solutions of double-sided QCQP with applications in signal processing. IEEE Trans. Signal Process. 62(5), 1093-1108 (2014)

27. H Li, P Stoica, J Li, Computationally efficient maximum likelihood estimation of structured covariance matrices. IEEE Trans. Signal Process. 47(5), 1314-1323 (1999)
28. M Steiner, K Gerlach, Fast converging adaptive processor or a structured covariance matrix. IEEE Trans. Aerosp. Electron. Syst. 36(4), 1115-1126 (2000)

29. A Aubry, A De Maio, L Pallotta, A Farina, Maximum likelihood estimation of a structured covariance matrix with a condition number constraint. IEEE Trans. Signal Process. 60(6), 3004-3021 (2012)

30. A Wiesel, Geodesic convexity and covariance estimation. IEEE Trans. Signal Process. 60(12), 6182-6189 (2012)

31. Z Luo, W Ma, A So, Y Ye, S Zhang, Semidefinite relaxation of quadratic optimization problems: From its practical deployments and scope of applicability to key theoretical results. IEEE Signal Process. Mag. 27(3), 20-34 (2010)

\section{Submit your manuscript to a SpringerOpen ${ }^{\circ}$ journal and benefit from:}

- Convenient online submission

- Rigorous peer review

- Immediate publication on acceptance

- Open access: articles freely available online

- High visibility within the field

- Retaining the copyright to your article

Submit your next manuscript at $>$ springeropen.com 